\title{
CHEESE-MAKING: SCIENTIFIC RESEARCH AND CONTROL
}

$\mathrm{T}$ HE oldest and one of the best ways of concentrating and conserving in a palatable form a large part of the nutritive value of milk is to make cheese of it. Whilst butter contains little more than the fat of the milk, cheese contains the casein, a large proportion of the valuable mineral salts and an important part of the vitamins as well. Only a relatively small proportion of the original water of the milk-approximately $2 \cdot 5-3$ per cent-remains in the ripened cheese, which if it is one of the more popular types of cheese in Great Britain, may be taken as containing about a third each of animal protein, fat and water. Cheese is probably, weight for weight, the most valuable of the ordinary foodstuffs in our dietary, since neither dried whole milk nor dried skim milk are purchased, nor readily purchasable, by the ordinary consumer in Great Britain. Approximately 200,000 tons of cheese, of which about twenty-five per cent was made in Great Britain, were consumed annually in this country before the War.

But there is cheese-and cheese. Unimpeachable organoleptic evidence exists for the statement that even under modern conditions cheese-making is not by any means a fully controlled industrial process. It is an art, as well as an "essay in applied biochemistry and bacteriology", and an art that sometimes breaks down seriously. Of late years, however, scientific research and control have made cheese manufacture less erratic.

At a joint meeting, under Mr. E. B. Anderson's chairmanship, of the Food Group of the Society of Chemical Industry and the Society of Public Analysts held in London on December 4, some aspects of recent research on cheese quality and its control were described by Prof. H. D. Kay and three of his colleagues of the National Institute for Research in Dairying. These investigations are a small part of an extensive programme of research on cheese-making and cheese quality that was getting under way at the Institute and at various co-operating cheese-making and other centres at the outbreak of war, and in which a fairly large team of research workers including a physicist, a statistician and a psychologist were collaborating. The War has inevitably interfered seriously with this plan; but a portion of the work, which has a direct bearing on cheese-making under war conditions, is still going on.

In an introduction by Prof. Kay this general plan was briefly outlined. Cheese is a complicated living system rather than a stable end-product, and a variety of techniques, chemical, biochemical, bacteriological, physical, physiological and psychological, are needed if the measurement and control of the many factors that go to make up cheese quality are to be satisfactorily effected. Methods for improved control of the quality of the original milk, of the cultures of lactic acid bacteria ('starters') added to promote acid production and flavour, of the enzymic characteristics of the rennet used, of the temperature and acidity of the milk and curd at various stages, of the timing of the succession of operations, of the physical properties of the curd and ripening cheese, are being considered. It is particularly necessary to define cheese quality so far as possible in precise terms, otherwise it cannot be consistently achieved.

Some recent findings on the nutritive value of various types of cheese were described in the introduction. Mr. J. Houston, who with Dr. S. K. Kon is investigating the proportion of the various vitamins of the original milk that remain in several staple varieties of cheese after different periods of ripening, has found that almost the whole of the vitamin $\mathrm{A}$ and carotene of the original milk remain in the cheese made from it, whether it is Cheddar, Cheshire or Stilton, and whether it is made in summer or winter.

It is not surprising that both these accessory factors, being fat-soluble, stay in the cheese, which contains practically the whole of the fat of the original milk; but it is of considerable significance that during the four to five months of the ripening process the conditions in the cheese are such that there is no destruction of either vitamin A or carotene.

Of the vitamin B group, preliminary experiments show that vitamin $B_{1}$ is lost in the whey to the extent of about nine tenths of that present in the original milk. Of the lactoflavin (riboflavin) distinctly less - only about three quarters-appears to go into the whey. On the supposition that these two vitamins are present in simple aqueous solution, it would be expected that more than 95 per cent of each would be lost. There is now little doubt, however, that a considerable proportion of each of these vitamins-particularly riboflavinis more or less firmly bound to the proteins of the milk which are clotted by rennet. About 10 per cent of the $B_{1}$ vitamin in the original milk, and about 25 per cent of the lactoflavin remain, there- 
fore, in the cheese, and suffer no serious change in amount during at least four months of ripening. If anything, Stilton contains a slightly larger proportion of the vitamins than the other two varieties.

Prof. Kay also brought forward some analytical work of Dr. E. C. V. Mattick by which she has shown that there is considerable variation in the content of mineral salts as between the commoner cheeses-Cheddar, Cheshire and Lancashire on one hand, and Stilton on the other. In the making of Stilton the curd is allowed to remain in contact with the whey for a longer time than in the case of the other three cheeses. As a consequence the Stilton curd is bathed in an increasingly acid medium (owing to the bacterial fermentation of the lactose which is still proceeding rapidly in the whey). As this increasingly acid medium slowly drains away it leaches out much of the calcium and phosphorus originally present in the curd. Whilst ripe Cheddar cheese, for example, contains 57 per cent of the calcium of the original milk and 50 per cent of the phosphorus, Stilton cheese of the same age and made from a portion of the same original bulk of milk only contains 7 per cent of the calcium and 26 per cent of the phosphorus. It is nutritionally of importance in war-time that each of the three most widely consumed cheesesCheddar, Cheshire and Lancashire-contains an equally high proportion of calcium. Stilton, a luxury cheese, is consumed for the most part by those whose diet is unlikely to be lacking in this mineral.

Flavour in cheese is bound up, to a large extent, with the presence of fatty acids, particularly the lower fatty acids, liberated during lipolysis. Miss E. R. Hiscox dealt with developments which she and her collaborators have introduced in the methods of determining volatile fatty acids in cheese, and with the significance of some of the recent findings obtained with the new technique.

Simple steam distillation of an acidified cheese mush gives results which are far too low, since both the fat and the protein of cheese hold back appreciable amounts of some of the volatile acids. Miss Hiscox gave details of a new method of analysis which depends on water extraction of the cheese mush, followed by extraction of the separated cheese fat with very dilute alkali, ether extraction of the residues and steam distillation of the acidified extracts. A particularly interesting finding is that the blue cheeses, Roquefort and Danish Blue, are in a different class from the blue cheeses Stilton and Gorgonzola as regards their content of fatty acids, and the behaviour of the fatty acids on extraction. The former pair are highest, Stilton and Gorgonzola lowest, in their volatile fatty acid content, whilst typical Cheddar and
Cheshire are intermediate. The correlation between these and other findings and flavour of the different cheeses was pointed out.

The chief chemical changes in ripening cheese are proteolysis, fermentation of the remaining lactose to lactic, acetic and propionic acids, and a certain degree of lipolysis. Dr. J. G. Davis described the part played by enzymes in these processes. He stressed the importance, from the point of view of cheese quality, of beginning with chemically normal (that is, mastitis-free) milk, and with rennet of good quality. Pasteurization (which destroyed most of the enzymes of the milk as well as a large majority of the micro-organisms) previous to cheese-making slows down ripening and prevents the attainment of the fullest flavour. It also prevents the development of certain taints and aids in the production of cheese of long keeping quality. Commercial rennet contains enzymes having at least four distinguishable activities: (a) clotting, (b) proteolysis of the pepsin type $(p \mathrm{H}$ optimum of 2), (c) proteolysis of the papain type (optimum $p \mathrm{H}$ about $4 \cdot 6),(d)$ proteolysis of the peptidase type (optimum $p \mathrm{H}$ about 6). Nevertheless, pure rennin free from pepsin produces equally good, if not better, cheese than commercial rennet.

Two of the enzymes which normally occur in raw milk-lipase and proteinase-are concerned in cheese-ripening. The latter produces a mellowing of the body of the cheese, owing to protein breakdown, but does not seriously affect the quantity of desirable flavour substances. Lipase, on the other hand, has an important effect on cheese flavour. It has recently been found that if small amounts of lipase are added to milk, a strong and slightly rancid flavour is produced in the cheese. This flavour, called by graders a "kexey" flavour, has been known for a long time, but its origin was not previously understood. Dr. Davis stated that cheese flavour is for the most part bound up with the lipolytic enzymes, which may be inherent in the original milk or of bacterial origin. The mild flavour of cheese made from pasteurized milk, or from very clean milk, can be correlated with the absence of bacterial lipase.

Quality in cheese was also dealt with, but from an entirely different angle, by Dr. G. W. Scott Blair. He pointed out the desirability of establishing objective criteria, both for controlling the various stages in the making of cheese and in the assessment of its final quality, rather than relying entirely on subjective personal judgment, though the latter could never be entirely dispensed with. He described new methods and apparatus, devised by himself and his colleagues at Shinfield, by which certain of the rheological properties of cheese and other products ean now be measured in c.G.s. 
units. One of these is a simple practical apparatus for deciding when curd shall be pitched (allowed to settle), the correct timing of which is highly important from the point of view of cheese quality. This apparatus, already in use in a few cheesemanufacturing centres, gives a numerical measure of the quality of the cut curd just before pitching time, which enables this time to be decided with some precision. Dr. Scott Blair showed also another simple apparatus, recently made for measuring the 'body' of ripening (or ripe) cheese, previously assessed literally by rule of thumb (pressure) or by the use of a special skewer. He described a rather more complicated apparatus now in use by which it is possible to maintain constant pressure per unit area on a sample of material the cross-section of which is increasing, and mentioned some of the unexpected results which cheese and a variety of materials have recently given when tested by it.

\title{
APPLICATION OF X-RAYS TO THE STUDY OF ALLOYS*
}

\author{
By DR. H. Lipson, \\ CaVendish Laboratory, Cambridge
}

$\mathrm{T}^{\mathrm{H}}$ HE use of X-rays has proved to be of fundamental importance in the investigation of alloy phase diagrams. The classical methodscooling curves and the microscopic examination of etched surfaces-are, of course, of immense value where the problem is simply that of the precise determination of phase boundaries, and it is probable that the use of $\mathrm{X}$-rays for this purpose is not of great importance. Where, however, more complex problems of equilibrium arise, the knowledge of crystal structures involved is essential.

As an example of this may be quoted the system aluminium-copper. Several investigators ${ }^{i}$ had recorded their views on that part of the system which lies between 15 and 30 per cent aluminium; but it was first found by X-rays ${ }^{2}$ that the problem was not one of ordinary phase equilibrium. The phases have all essentially the same crystal structure, and vary only in the way in which the two sorts of atoms are arranged on the positions available. This results in changes of symmetry ${ }^{3}$ which are not ordinary phase changes and which do not produce regions of two-phase equilibrium. Thus the problem is not one of which the microscope could be expected to produce a solution.

For the investigation of this type of structural change it is necessary to use apparatus of high resolving power. As in certain types of optical interferometer, this is achieved by using the high orders of interference, particularly those which are scattered back almost into the incident X-ray beam. Since, however, the deviation of a particular order is fixed by the dimensions of the unit cell and the wave-length of the radiation, the only

* Many references are made to the paper by Bradley, Bragg and Sykes $(J$. Iron and Steel Inst., 141, $63 ; 1940)$ as well as to original papers. This is referred to in the footnote references as 'B.B.S.' followed by the page number of the particular item. The diagrams are also from this paper. control one has over the resolution lies in the dimensions of the apparatus. Increasing distances decrease the intensity of the diffracted beams, and so the advantage of increased resolution has to be balanced against the disadvantage of increased exposure time. Progress has therefore been dependent on the improvement of $\mathrm{X}$-ray tubes and $X$-ray film, and at the present day cameras can be used which would have been considered of fantastic size only ten years ago. At Prof. W. L. Bragg's laboratory in Manchester in 1924 a camera of diameter $2.5 \mathrm{~cm}$. was used, followed by one of $5 \mathrm{~cm}$. In 1926 a 'large' camera of $9 \mathrm{~cm}$. diameter was introduced and was used to find the structure of $\gamma$-brass ${ }^{4}$. This camera was a standard instrument for many years, and in 1934 a still larger one of $19 \mathrm{~cm}$. diameter was made and was used for special problems ${ }^{5}$. At Cambridge this has now become the standard instrument, and a camera of $35 \mathrm{~cm}$. has also been used with some success ${ }^{8}$. With this camera it is probable that the limits of resolution for the high orders have been reached, but still larger ones may be necessary for separating the multitude of lines at low angles given by complex structures.

\section{Phase Diagrams}

Although the most important function of $\mathrm{X}$-rays must always be the determination of crystal structures, a great deal of valuable information has been obtained about the equilibrium, and approach to equilibrium, of many alloy systems. This information merely adds to the vast amount already obtained by the standard methods of metallurgy; but it is data which would be obtainable in no other way. The reason for this is two-fold. First, each phase gives its own characteristic X-ray 